Musées, Patrimoine et Culture scientifiques et techniques

$135 \mid 2011$

mai - juin 2011

\title{
Impliquer un Conseil scientifique dans les décisions de demain
}

Getting the Scientific Council to be involved in tomorrow's decisions

\section{Pauline Burnel}

\section{OpenEdition \\ Journals}

Édition électronique

URL : http://journals.openedition.org/ocim/866

DOI : 10.4000/ocim.866

ISSN : 2108-646X

Éditeur

OCIM

Édition imprimée

Date de publication : 1 mai 2011

Pagination : 13-18

ISSN : 0994-1908

Référence électronique

Pauline Burnel, «Impliquer un Conseil scientifique dans les décisions de demain », La Lettre de l'OCIM

[En ligne], 135 | 2011, mis en ligne le 01 mai 2013, consulté le 19 avril 2019. URL : http://

journals.openedition.org/ocim/866 ; DOI : 10.4000/ocim.866

Tous droits réservés 


\section{Le Vaisseau : un équipement unique}

Le Vaisseau est un centre de découverte des sciences et des techniques destiné aux enfants de 3 à 15 ans, initié par le Conseil général du Bas-Rhin. À travers cette mission éducative, le Vaisseau s'attache à tenir une promesse : "apprendre en s'amusant », en donnant aux enfants l'occasion de développer leur curiosité pour les sciences par une approche ludique : jeu, manipulation, action, création, expériences dans des petits laboratoires de la vie, qui se visitent avec les cinq sens. Le Vaisseau épaule les enseignants et les parents dans leur mission éducative et peut bien sûr susciter des vocations scientifiques et techniques.

Un certain nombre de valeurs sont ainsi intégrées aux actions du Vaisseau, parmi lesquelles :

- l'attention portée aux enfants,

- le partage de la découverte et la coéducation,

- l'ouverture sur d'autres cultures et notamment rhénane,

- laction en faveur des personnes en situation de handicap,

- le respect de l'environnement.

Le Vaisseau est en régie directe, avec un budget de 5 millions d'euros autofinancé à hauteur de $33 \%$ (billetterie, mécénat, recettes de la boutique ou encore de la cafétéria...). Avec plus d'un million de visiteurs accueillis et plus de $2000 \mathrm{~m}^{2}$ d'exposition, le Vaisseau est un équipement unique en région. Depuis l'année dernière, il coproduit même des expositions : l'une avec la Cité des Sciences et de l'Industrie à Paris, Grains de bâtisseurs présentée dès la rentrée 2010, d'autres avec Cap Sciences à Bordeaux autour d'une thématique sur le vent, la nature et l'homme, programmée pour 2011 ou encore Plantastic réalisée avec d'autres acteurs européens dont le National Museum à Liverpool pour 2012. Dès sa phase projet, le Vaisseau a fait le choix de se faire accompagner par une instance consultative réunissant notamment des experts issus des domaines de la médiation, des sciences et des techniques. Cette instance consultative est nommée : le Conseil scientifique et pédagogique du Vaisseau.

\section{Le Conseil scientifique : un moteur de performance...}

\section{Le processus de décision au Vaisseau}

Le Vaisseau a 4 missions fondamentales inscrites dans les principes de sa programmation culturelle depuis son ouverture :

- rendre la science accessible au plus grand nombre,

- participer à l'éducation,

- stimuler l'ouverture au monde,

- être acteur du développement durable.

Au-delà de ses missions fondamentales, le Vaisseau développe un projet d'établissement sur plusieurs années définissant les grands axes de développement de l'équipement, validé par le président du Conseil général. Le projet d'établissement 2008-2012 est le fruit d'une concertation avec l'ensemble de l'équipe du Vaisseau et les élus en charge du Vaisseau. Mené par la direction, il se décline ensuite en actions ou actions prioritaires par service. Les actions menées chaque année s'inscrivent donc dans ce cadre général et sont régulièrement soumises pour avis à l'une des instances consultatives du Vaisseau.

\section{La place du Conseil}

Le Conseil scientifique et pédagogique du Vaisseau est l'une des trois instances consultatives dont dispose le Vaisseau, les deux autres sont la Commission de la jeunesse et le Conseil consultatif et de développement (voir tableau ci-dessous).

\begin{tabular}{|l|l|l|}
\hline Nom & Champs d'intervention & Exemple de sujets traités \\
\hline $\begin{array}{l}\text { Commission de la jeunesse } \\
\text { du Conseil général }\end{array}$ & $\begin{array}{l}\text { Émet des avis sur tous les sujets soumis } \\
\text { à décision des instances délibérantes du } \\
\text { Conseil général (commission permanente, } \\
\text { session plénière) }\end{array}$ & $\begin{array}{l}\text { - Budget } \\
\text { - Tarification } \\
\text { - Grands principes } \\
\text { de programmation culturelle }\end{array}$ \\
\hline $\begin{array}{l}\text { Conseil consultatif } \\
\text { et de développement } \\
\text { du Vaisseau }\end{array}$ & $\begin{array}{l}\text { - Analyse de l'activité (offres culturelles) } \\
\text { - Résultats de gestion au regard des } \\
\text { objectifs assignés par le Conseil général } \\
\text { - Orientations stratégiques } \\
\text { - Suivi et mise en ouvre des décisions stratégiques }\end{array}$ & $\begin{array}{l}\text { - Budget 2009 et perspectives } \\
\text { budgétaires 2010 } \\
\text { - Renouvellement de la scénographie } \\
\text { de l'exposition permanente }\end{array}$ \\
\hline $\begin{array}{l}\text { Conseil scientifique } \\
\text { et pédagogique } \\
\text { du Vaisseau }\end{array}$ & $\begin{array}{l}\text { Orientations stratégiques, notamment en } \\
\text { matière de contenu scientifique, médiation } \\
\text { scientifique et pédagogique }\end{array}$ & $\begin{array}{l}\text { - Renouvellement de la scénographie } \\
\text { de l'exposition permanente } \\
\text { - Introduction de l'objet industriel }\end{array}$ \\
\hline
\end{tabular}

Les trois instances consultatives du Vaisseau 
La Commission de la jeunesse et le Conseil consultatif et de développement réunissent des représentants du Conseil général du Bas-Rhin plus particulièrement en charge des grandes orientations de gestion et de stratégie du Vaisseau.

Le Conseil consultatif et de développement du Vaisseau est saisi pour avis facultatif en amont de la Commission thématique à laquelle est rattaché le Vaisseau (appelée Commission de la jeunesse). Dans ce cadre, il est notamment appelé à se prononcer sur :

- l'analyse de l'activité, essentiellement en termes d'offres culturelles ;

- les résultats de la gestion tant passée (comptabilité analytique) que future (simulations budgétaires faites selon l'échéancier du projet d'établissement en cours), au regard des objectifs assignés par le Conseil général ;

- les orientations stratégiques à proposer au Conseil général ;

- le suivi de la mise en œuvre des décisions stratégiques.

Le Conseil scientifique et pédagogique émet des avis externes sur les grandes orientations de l'équipement notamment en matière de contenu scientifique, de médiation scientifique et de pédagogie.

Les avis du Conseil scientifique et pédagogique sont systématiquement communiqués aux membres de la Commission thématique du Conseil général du BasRhin en charge du Vaisseau (Commission de la jeunesse). Il arrive que certains sujets soient d'abord présentés au Conseil consultatif et de développement (voir le schéma ci-dessus).

Les avis et appuis des personnalités du Conseil scientifique et pédagogique permettent d’asseoir la réputation

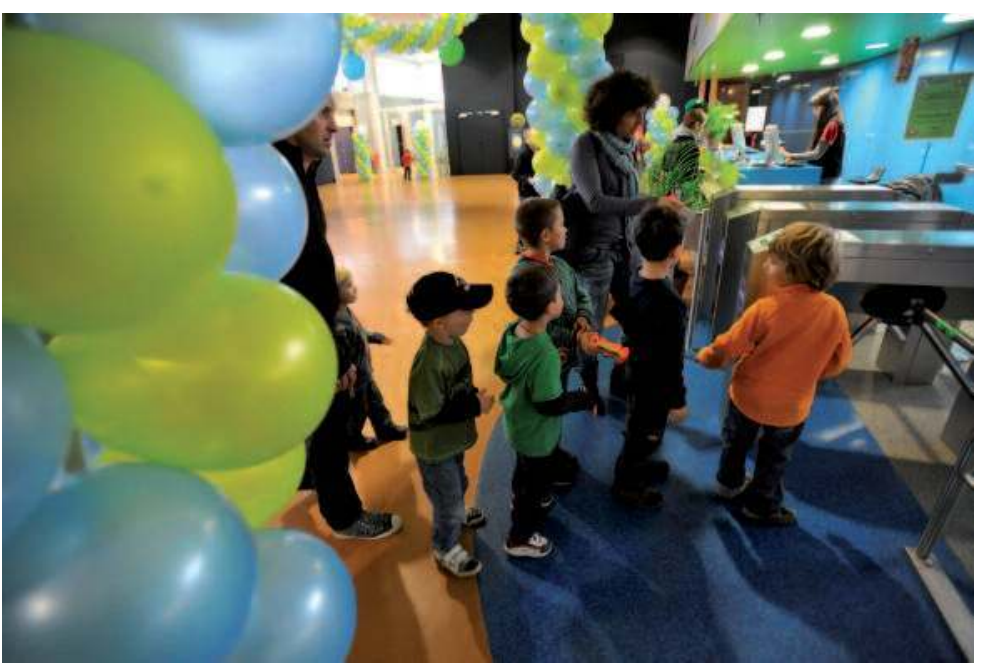

Accueil des visiteurs, le jour du 1000000 e visiteur du Vaisseau ๑ J.L. Stadler

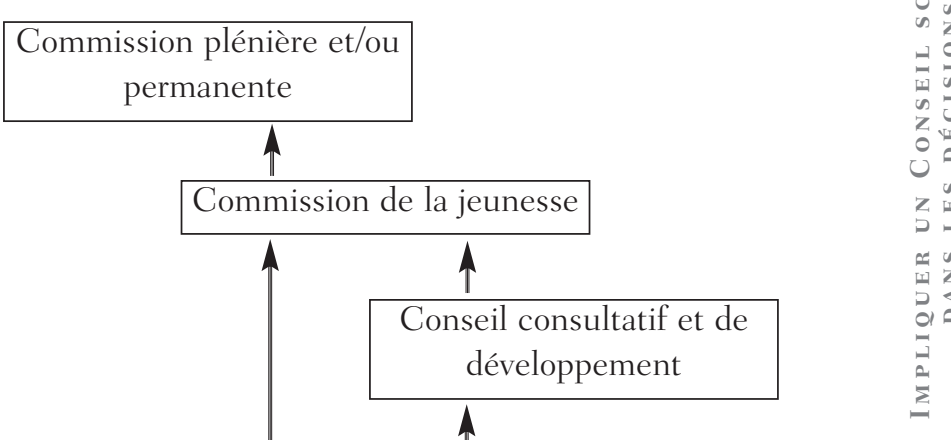

et le sérieux des activités développées par le Vaisseau et constituent un efficient réseau de partenaires.

\section{La composition du Conseil}

Le Conseil, nommé par décision officielle du président du Conseil général, doit rassembler des compétences adaptées aux enjeux de l'équipement. Ainsi, le Conseil scientifique et pédagogique du Vaisseau présidé par Marie-Christine Creton - ancienne directrice de l'INSA Strasbourg - est composé de 23 membres représentatifs de l'activité de l'équipement strasbourgeois : élu du Conseil général, universitaires, directeurs de centres de sciences nationaux, européens ou internationaux, inspecteur de l'Éducation nationale, enseignants et pédagogues, représentants de la société civile (journalistes, membre de la chambre de consommation ${ }^{(1)}{ }^{\ldots}$.).

Une telle diversité n'est pas commune. Pour autant, nos concitoyens - et donc la société civile - sont demandeurs de repères et souhaitent s'impliquer de plus en plus dans la CSTI. La science n'est pas une seule question de spécialistes, elle est une question de société qui engage notre responsabilité individuelle et collective. C'est pourquoi le Vaisseau souhaite mobiliser l'ensemble des acteurs, dont des journalistes qui ont aussi la mission de vulgariser la science.

Outre une pluridisciplinarité (variétés des métiers et des expériences), une autre particularité du Conseil scientifique du Vaisseau est sa composition pluriculturelle. Jouissant d'un emplacement géographique particulier (à un kilomètre de la frontière allemande) et de la vocation européenne et transfrontalière de la ville de Strasbourg, le Vaisseau n'est pas un projet franco-français, par conséquent les membres de son 


\section{... Mais aussi un défi réel}

Impliquer et animer un Conseil scientifique est un privilège (recevoir un soutien ou un avis d'experts sur le développement de son équipement), mais également un défi réel : il s'agit de veiller à ce que les avis ou recommandations émis par le Conseil scientifique soient applicables (pour l'équipement, le visiteur, l'équipe...). La diffusion d'une information structurée comme évoquée auparavant est nécessaire mais non suffisante : il s'agit aussi de faciliter les rencontres sur site, d'établir une prise de contact directe, favoriser l'échange.

Définir les conditions idéales de fonctionnement d'un Conseil est une préoccupation importante. Le Vaisseau s'y est bien sûr intéressé et a par exemple constaté que les ordres du jour des précédents Conseils scientifiques étaient trop denses et ne permettaient pas aux membres d'explorer le Vaisseau et de découvrir les nouveautés (expositions, éléments, animations). Le contenu des rencontres semestrielles a donc été récemment modifié pour offrir aux membres une phase d'observation du Vaisseau et de ses visiteurs. Cette phase favorise la connaissance de l'équipement d'une part, mais aussi l'objectivité d'autre part (les membres constatent par eux-mêmes, interrogent les visiteurs...).

Les membres eux-mêmes ont été invités à s'exprimer sur la qualité du Conseil scientifique, à évaluer son fonctionnement. Encore une fois, il ne s'agit pas d'un simple recueil par voie de questionnaire mais un échange basé sur des questions ouvertes. À l'issue de ce dernier, plusieurs pistes d'amélioration sont apparues parmi lesquelles :

- la possible présence d'un spécialiste externe ou expert de la thématique traitée qui permettrait d'améliorer la contribution du Conseil, d'enrichir le débat ; - un temps dédié aux membres pour exposer une particularité de leur établissement et ainsi mieux se connaître ;

- ou encore la mise en place d'un outil collaboratif leur permettant d'échanger en dehors des rencontres semestrielles proposées.

Ces nouvelles « règles » seront effectives dès les prochaines rencontres. Elles répondent bien à un souci d'applicabilité des conseils émis par les membres: expérimenter, observer, c'est mieux connaître l'équipement, ses particularités et ses visiteurs, et donc mieux répondre aux enjeux de son développement.

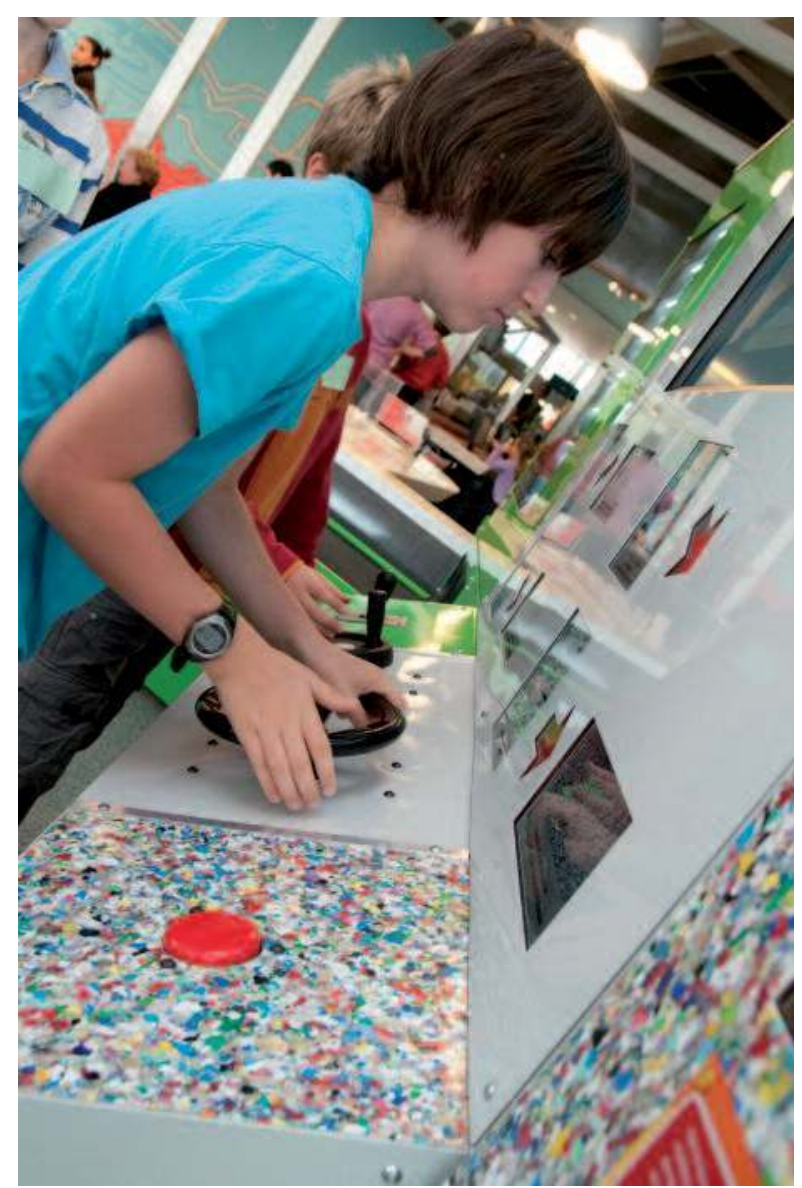

Le Vaisseau : atelier « Les bouteilles en plastique, ça se recycle ! Univers Je fabrique »
(c) Cg67

\section{Une relation nouvelle entre Conseil scientifique et équipe du Vaisseau}

Les membres du Conseil sont particulièrement impliqués dans les décisions du Vaisseau. Au-delà d'un intérêt, d'une posture d'écoute et d'échange, un élément détermine le succès d'une relation : le suivi des idées et conseils émis. Il apparaît très clairement que de savoir comment les idées et conseils émis sont utilisés par le Vaisseau est un facteur de motivation. Le suivi des idées repose sur trois conditions :

- une utilisation simple,

- des contributions reconnues,

- un potentiel optimisé.

Ainsi, le Vaisseau utilise des thématiques traitées en Conseil scientifique pour des réflexions internes dans le but de l'approfondir. C'est le cas par exemple du sujet « 8 animateurs-concepteurs, 180 éléments interactifs : quel dosage entre médiation humaine et médiation 
mécanique ? " traité par le Conseil scientifique et

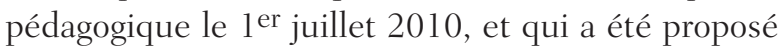
à l'équipe du Vaisseau sous forme de comité de réflexion à l'automne de la même année : «Présence humaine en salle : quel temps hors public et face public pour le personnel pour garantir une meilleure médiation humaine? ».

Si la principale feuille de route du Vaisseau reste son projet d'établissement, un document mené par la direction du Vaisseau fixant les axes de développement de l'équipement sur une période de 4 ans, cette feuille de route est régulièrement confrontée aux regards externes des instances consultatives dont le Conseil scientifique fait partie. Un mécanisme fructueux qui libère le questionnement, l'étude, l'approfondissement et garantit par conséquent toute la qualité pédagogique du Vaisseau.

\section{Synthèse des éléments}

favorisant l'efficacité d'un Conseil

- Une palette de compétences et d'expériences

- Un ordre du jour co-construit

- Une information soigneusement structurée

- Une place prépondérante à l'échange

- L'organisation de débats productifs

- À l'issue de chaque rencontre, l'édition d'un mémo des avis émis

Une chose est sûre, le travail des uns et des autres participe à un processus d'amélioration continu pour le Vaisseau.

Là où il n'y a pas de formule unique, certains facteurs sont pourtant facilitateurs : la variété des expériences et des points de vue, la liberté des sujets, l'interactivité. Et la fidélité des membres, pour le Vaisseau, en est certainement le premier indicateur de réussite.

\section{Note}

(1) La Chambre de Consommation d'Alsace (CCA) fédère près d'une vingtaine d'associations consuméristes régionales et vise notamment à anticiper l'évolution des comportements et accompagner les consommateurs vers des modes de consommation plus responsables. Elle a donc un rôle de médiation visant à rapprocher le consommateur du producteur ou de l'industrie, ce que le Vaisseau fait également, sur un terrain scientifique. 